\title{
Research on the estimation method of the point-of-interest (POI) displacement for ultra-precision flexible motion system based on functional optical fiber sensor
}

\author{
Yujie $\mathrm{Li}^{1,2}$, Ming Zhang ${ }^{1,2,}$, and $\mathrm{Yu} \mathrm{Zhu}^{1,2}$ \\ ${ }^{1}$ State Key Lab of Tribology, Tsinghua University, Haidian District, Beijing 100-084, China \\ 2 Beijing Key Lab of Precision/Ultra-precision Manufacturing Equipments and Control, Tsinghua University, Haidian District, \\ Beijing 100084, China
}

Received: 20 January 2021 / Accepted: 22 October 2021

\begin{abstract}
This paper proposes a POI displacement estimation method based on the functional optical fiber sensor and the phase modulation principle to improve the POI displacement estimation accuracy. First, the relation between the object deformation and the optic fiber lightwave phase is explained; the measurement principle of functional optical fiber sensor based on the heterodyne interference principle and its layout optimization method is proposed, and a POI displacement estimation model is presented based on the data approach. Secondly, a beam is taken as the simulation object, the optimal position and length of the optical fiber sensor are determined based on its simulation data. Finally, the experimental device is designed to verify the effectiveness of the POI displacement estimation method based on the optic fiber sensors. The frequency-domain plot of the signals shows that the optical fiber sensors can express the flexible deformation of the analyzed object well. The POI displacement estimation model with the fiber optic sensor signals as one of the inputs is constructed. Through estimating the test data, the error using the optical fiber sensor-based POI displacement estimation method proposed in this paper reduces by more than $61 \%$ compared to the rigid body-based assumption estimation method.
\end{abstract}

Keywords: Functional optical fiber sensor / point-of-interest (POI) / heterodyne interference principle / layout optimization / POI displacement estimation model

\section{Introduction}

High-performance motion systems, such as the wafer stage in the semiconductor industry continuously, call for higher throughput and accuracy, resulting in more challenges for the design in next-generation motion stages [1]. Meanwhile, these increasingly stringent performance requirements result in a desire for increased acceleration and control performance [2]. The increased acceleration implies a more lightweight system design or higher actuation forces, causing that the flexible dynamic behavior is more prominent or even appear at lower frequencies [3-7]. A higher control bandwidth helps improve the control performance of the system, but meanwhile, it will aggravate the flexibility characteristics. The non-negligible flexibility will lead to the displacement gap between the point of interest and the point of control to become larger. The feedback displacement obtained based on the

\footnotetext{
* e-mail: zhangm@Tsinghua.edu.cn
}

rigid-body assumption will not meet the system control requirements [8]. The point-of-control (POC) displacement as the input of the controller can be the displacement of the measurement point or the displacement calculated by measurement points. The point of interest is the system performance point. Its control performance determines the system performance. So, the POI displacement should be essentially used as the input of the controller. However, due to the various constraints of the system structure, the POI displacement cannot be used as the input of the system feedback. In the lithograph system [9-11], as shown in Figure 1, the sensors are located at the edge of the wafer stage due to the structural design. The points of interest are located in the exposure area of the wafer stage $[9,12]$, and the POC displacements are calculated by measurement points. Therefore, it is necessary to propose a method to improve the POI displacement estimation accuracy in ultra-precise motion systems with flexibility.

Common methods of POI displacement estimation mainly include model-based and data-based methods. The model-based methods mainly estimate POI displacement 


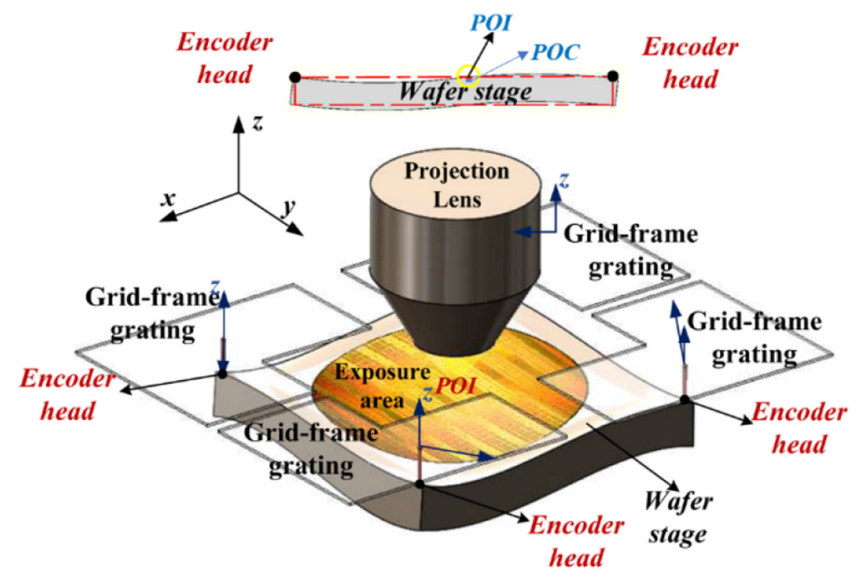

Fig. 1. POC and POI in the schematic of the flexible wafer-stage system.

by constructing observers [13-15]. Observers usually include the undisturbed observers $[13,14]$ and the disturbance observers [15-17]. The undisturbed observer only consists of the controlled object itself, while the disturbance observer is composed of the controlled object and the disturbance model. In reference [13], the importance of the POI displacement estimation using the observer was evaluated from the positioning accuracy of the system through simulation analysis; the paper [14] analyzes the importance of the POI displacement estimation using the observer from the comparison of the magnitude of the flexibility of the closed-loop transfer function by building a single-degree-of-freedom experimental setup and introducing active control; the paper [15] illustrates the effectiveness of the POI displacement estimation using observer by comparing the amount of output information without the observer and with the observer. The advantage of this method is that the internal state change mechanism of the system can be understood through the observer construction process. Simultaneously, because it is highly dependent on the model, the accuracy of the model determines the accuracy of the POI displacement estimation. Usually, the disturbance model is unpredictable and stochastic. It is mainly constructed by the equivalent method based on engineering experience, such as the airflow disturbance is equivalent to Brownian motion. However, the deviation caused by equivalence, unpredictability, and randomness can lead to the inaccuracy of the disturbance observer model and thus affect the accuracy of the POI displacement estimation. The modeling methods of the controlled objects mainly include the finite element modeling method [16] and the system identification method $[14,18]$. For the ultraprecise motion system with flexible characteristics, the complexity of the model increases, and the difficulty of identification increases accordingly. The identification model acquired based on the existing sensors and the corresponding layout information can no longer meet the actual control requirements. For example, in the literature [18], 16 additional local sensors are added to obtain more details of the model, improving the identification accuracy.

Compared with the model-based approach, the databased approach starts directly from the available data of the system. This data itself contains information about the structural changes, unmodeled dynamics and unknown disturbances of the system. This approach gets rid of the dependence on the controlled object model. Thus, the robustness of the estimation accuracy can be improved. In the literatures $[10,19,20]$, the layout of the existing limited number of sensors is optimized, the linear least-squares method is used to obtain the mapping matrix between the measured and desired points, and the cloud map of estimation errors is reduced from tens of nanometers to the hundred picometer level by simulation analysis. The goal of the investigated literature is to propose an enlightening mathematical method, but there is a certain distance from practical engineering applications. Similar to the modelbased method, there will be incomplete sample data for high-precision systems with prominent flexibility in practice. In the literature [10], the amount of sensor output information increases by optimizing the sensor layout, but for example, the measurement sensors in the wafer system mainly include laser interferometers or plane gratings [21]. Limited by the installation space and the volume of the sensor itself, these sensors can only be located on the edge of the wafer stage, and the number of the sensors cannot be added arbitrarily. When the flexible characteristics of the structure are continuously excited, the dynamic deformation of the structure becomes more complicated, and the amount of information transmitted by the high-precision displacement sensor is not enough to reflect the POI displacement.

Therefore, whether based on model-based [18] or databased approach $[10,19]$, for ultra-precise motion systems with prominent flexibility characteristics, it is necessary to increase the output of the model information, ensuring the accuracy of model identification and the completeness of model output data. The local compact vibration sensors attached to the surface of the structure can be added to obtain structural flexibility information. Acceleration and resistance strain gauges are often used for vibration. For example, in references $[22,23]$, the specific points in the measurement bridge where the sensors cannot be installed are used to obtain the vibration information of the measured object by fusing acceleration sensors and resistive strain gauges, and the laser displacement sensors are used as reference sensors to achieve the estimation of the unmeasurable points. In reference [24], to improve the control accuracy, the motion displacement of a flexible beam is obtained through the resistance strain gauge, acceleration sensor and gyroscope as the system feedback inputs. However, the local sensors in such applications exhibit low measurement accuracy and are not suitable for the ultra-precise motion systems. The optical sensor as a local sensor is with high-resolution characteristics in addition to being small and basically free from structural constraints. The functional optical fiber sensor combined with the phase modulation principle has a resolution of $0.01 \mu \varepsilon[25,26]$.

According to the above review, this paper proposes a POI displacement estimation method for ultra-precision motion systems based on the functional optical fiber sensors and the phase modulation principle. The main contents are as follows: Section 2 presents the specific measurement principle based on this measurement scheme 
and the layout optimization of the functional optical fiber sensor. Then, a POI displacement estimation model is proposed using a time-series dynamic neural network based on the data approach. In Section 3, the optimal optical fiber sensor positions and lengths corresponding to the critical modes are determined using the simulation data of the controlled object by the proposed sensor layout optimization method. In Section 4, based on the above contents, an experimental setup is designed, and a POI displacement estimation model is constructed based on the optical fiber sensor output data using a data-based approach. The validity of the POI displacement estimation model based on the optical fiber sensor is proved through comparative analysis.

\section{Principle}

\subsection{Optical fiber sensor selection and measurement principle}

The design of the measurement scheme based on the optical fiber sensor revolves around two aspects: the selection of the sensor and the measurement principle.

\subsubsection{Sensor selection}

In the ultra-precision motion system, the positioning accuracy is at the nanometer level. Therefore, in terms of sensor selection, a functional optic fiber sensor is selected from a high-resolution perspective [25,26]. The functional sensor uses the characteristics of the optical fiber itself as a sensitive element to modulate the light transmitted within the optical fiber, making the intensity, phase, frequency or polarization state of the transmitted light change, and then the modulated signal is demodulated to obtain the measured signal $[27,28]$.

\subsubsection{Measurement principle}

The phase modulation method is widely used in optical fiber sensing fields such as fiber-optic sensors and fiber-optic hydrophones for its simple hardware structure and easy multiplexing [29]. The basic sensing mechanism of the phasemodulated optic fiber sensor is to change the phase of the light wave propagating in the optical fiber by the action of the measured energy field, and then use interferometric techniques to convert the phase transformation into a change in light intensity to detect the physical quantity to be measured.

When the measured object undergoes flexible vibration, the length and longitudinal direction (axial direction) of the measured object are subjected to mechanical stress. In the modal coordinate, it is characterized by the following relation:

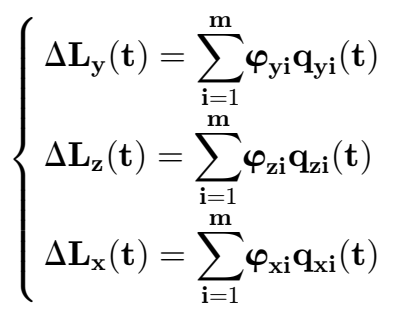

where $\Delta L_{(x, y, z)}(t)$ the change in three directions; $x$ is the longitudinal direction, $y$ and $z$ is the transverse direction.
Moreover, the corresponding $i$ th eigenvector and modal displacement are $\phi_{(x i, y i, z i)}, q_{(x i, y i, z i)} ; m$ denotes the measured object has $m$ modes.

When an optical fiber receives mechanical stresses in the longitudinal (axial) direction, the length, core diameter, and core refractive index of the optical fiber will change, and these transformations will lead to the change in the phase of the optical wave in the optical fiber. The specific expression is [28]:

$$
\Delta \boldsymbol{\phi}=\boldsymbol{\beta} \Delta \mathbf{L}+\Delta \boldsymbol{\beta} \mathbf{L}=\boldsymbol{\beta} \mathbf{L} \frac{\Delta \mathbf{L}}{\mathbf{L}}+\mathbf{L} \frac{\partial \boldsymbol{\beta}}{\partial \mathbf{a}} \Delta \mathbf{a}+\mathbf{L} \frac{\partial \boldsymbol{\beta}}{\partial \mathbf{n}} \Delta \mathbf{n},
$$

where $\beta=2 \pi / \lambda$ is the propagation constant of light waves in optical fiber, $\lambda=\lambda 0 / n$ is the propagation wavelength of light waves in optical fiber, $\lambda_{0}$ is the propagation wavelength of light waves in vacuum, $n$ is the refractive index of light waves, and $a$ is the radius of the optical fiber core. In this formula, the first term is the strain effect, which indicates the phase delay caused by the change of the optical fiber; the second term is the Poisson effect, which is the phase delay caused by the change of the optical fiber radius; the third term is the elasto-optical effect, which represents the phase delay caused by the change of the induced refractive index.

For the single-mode fiber, the strain optical matrix and Hooke's law are introduced into equation (2), and the general form of the single-mode fiber can be obtained [28]:

$$
\begin{aligned}
& \Delta \boldsymbol{\phi}=\mathbf{n} \frac{2 \boldsymbol{\pi}}{\boldsymbol{\lambda}_{0}} \mathbf{L} \\
& \left(1-\frac{1}{2} \mathbf{n}^{2}\left((1-\mathbf{v}) \mathbf{P}_{12}-\mathbf{v} \mathbf{P}_{11}\right)-\mathbf{v}\left(\frac{\boldsymbol{\lambda}}{4 \mathbf{a}}\right)^{2} \frac{1}{\left(1-\left[\frac{\boldsymbol{\lambda}}{4 \mathbf{a}}\right]^{2}\right)^{0.5}} \frac{1}{2 \mathbf{a}}\right) \boldsymbol{\varepsilon}_{3},
\end{aligned}
$$

where $P_{11}$ and $P_{12}$ are the strain optical constants; $\varepsilon_{3}=\Delta L /$ $L$ is the longitudinal strain of the optical fiber; $v=\mid \varepsilon_{1} / \varepsilon_{3} /$ is the Poisson's ratio, and $\varepsilon_{1}$ is the transverse strain of the optical fiber.

Combining equations (1) and (3), the relationship between the phase change and the eigenvalue of the object and the modal displacement can be obtained:

$$
\begin{gathered}
\Delta \boldsymbol{\phi}=\mathbf{n k}_{0} \\
\left(1-\frac{1}{2} \mathbf{n}^{2}\left((1-\mathbf{v}) \mathbf{P}_{12}-\mathbf{v} \mathbf{P}_{11}\right)-\mathbf{v}\left(\frac{\boldsymbol{\lambda}}{4 \mathbf{a}}\right)^{2} \frac{1}{\left(1-\left[\frac{\boldsymbol{\lambda}}{4 \mathbf{a}}\right]^{2}\right)^{0.5}} \frac{1}{2 \mathbf{a}}\right) \\
\sum_{\mathbf{i}=1}^{\mathbf{m}} \boldsymbol{\varphi}_{\mathbf{x i}} \mathbf{q}_{\mathbf{x i}}(\mathbf{t}) .
\end{gathered}
$$

Interferometric techniques, including homodyne interferometry and heterodyne interferometry, are often used to obtain the phase change. The homodyne interferometry technique is susceptible to low-frequency electronic noise, while the heterodyne interferometry technology can avoid the interference of low-frequency electronic noise through modulation and demodulation. From the perspective of anti-interference, the heterodyne interference is selected to obtain the phase change of the optical fiber. 
The two coherent light beams are with the frequencies $f_{1}$ and $f_{2}$, the light vector amplitudes $E_{1}$ and $E_{2}$, and the initial phases $\theta_{1}$ and $\theta_{2}$. When the object vibration causes the optical fiber to be stretched or compressed in the measurement path, the optical path difference is generated in the optical fiber. It means that the phase change of the light vector $\Delta \phi(t)$ is introduced into the measurement light. Then the light vectors of the measured light and the reference light can be expressed as:

$$
\left\{\begin{array}{l}
\mathbf{E}_{\text {ref }}=\mathbf{E}_{1} \mathbf{e}^{\mathbf{i}\left(\boldsymbol{\theta}_{1}+2 \pi \mathbf{f}_{1} \mathbf{t}\right)} \\
\mathbf{E}_{\text {mea }}=\mathbf{E}_{2} \mathbf{e}^{\mathbf{i}\left(\boldsymbol{\theta}_{2}+2 \pi \mathbf{f}_{2} \mathbf{t}+\Delta \boldsymbol{\phi}(\mathbf{t})\right)} .
\end{array}\right.
$$

Two light waves interfere in the optical fiber, and the light intensity at a point in the interference field can be expressed as:

$$
\begin{aligned}
\mathbf{I} & =\left(\mathbf{E}_{\text {ref }}+\mathbf{E}_{\text {mea }}\right)\left(\mathbf{E}_{\text {ref }}+\mathbf{E}_{\text {mea }}\right) \\
& =\mathbf{E}_{1}^{2}+\mathbf{E}_{2}^{2}+2 \mathbf{E}_{1} \mathbf{E}_{2} \cos \left(\boldsymbol{\theta}_{1}-\boldsymbol{\theta}_{2}-\Delta \boldsymbol{\phi}(\mathbf{t})+2 \boldsymbol{\pi} \Delta \mathbf{f} \mathbf{t}\right),
\end{aligned}
$$

where $\Delta f=f_{1}-f_{2}$.

Combining equations (4) and (6), the relations among the POI eigenvectors, the phase change and the optical intensity of the fiber light wave can be seen:

$$
\phi_{\mathrm{xi}} \propto \Delta \phi \propto I,
$$

As mentioned above, the phase change is proportional to the strain eigenvector and the modal displacement, meaning that the optical fiber change can characterize the flexible properties of the object.

In summary of the above measurement principles, a frequency synthesizer and two acousto-optic modulators are utilized to complete the acquisition of signals from the optical fiber sensors based on the principle of heterodyne interferometry [30]. First, the light wave from the laser is divided into two by an optical splitter, and the two signals with a certain frequency difference are formed through the frequency synthesizer and the acousto-optic modulator. One is the reference path, and the other is the measurement path. After the reference signal interferes with the measurement signal, the phase information is obtained through the phase board. The specific measurement principle is shown in Figure 2.

\subsection{Layout optimization of optical fiber sensor}

Unlike the point measurement of common sensors, the functional optical fiber sensor measurement is a line measurement. In modal coordinates, the flexible displacement of the analyzed object can be described as the $m$-order modal superposition. It is necessary to find several critical modes contributing the most to the flexible displacement. Therefore, a sensor optimal layout method with this measurement feature, including optimizing the location and length of the sensor based on critical modes of the measured object in the interest area, is proposed. The details are as follows:

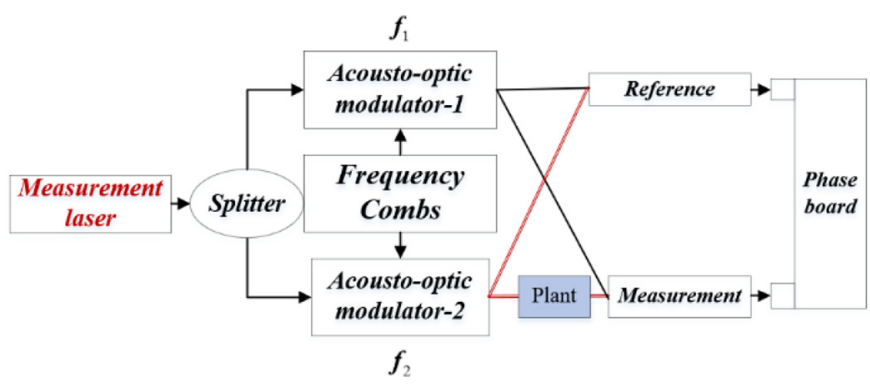

Fig. 2. Schematic of the functional optical fiber sensor measurement principle.

(1) Judgment of critical modes in the interest area

When the structural flexibility is excited, every POI exhibits different dynamic characteristics. The critical modes are determined in the interest area with the orientation of displaying the POI displacement.

The proportion of the motor-driven input corresponding to each mode is set under certain excitation condition:

$$
\left(\mathbf{p}_{\mathbf{f}}\right)_{\mathbf{i}}=\frac{\mathbf{f}_{\mathbf{i}}}{\sum_{\mathbf{i}=1}^{\mathbf{m}} \mathbf{f}_{\mathbf{i}}}
$$

where, for each mode, $f_{i}$ represents the $i$ th modal force, and $\left(p_{f) i}\right.$ is the percentage of the $i$ th modal force.

It is assumed that the longitudinal deformation of the POI in the interest area is $\Delta L$. The interest area and frequency bands are pre-defined. The deformation corresponding to the critical mode is determined under the conditions of the specific excitation conditions in equations (9) and (10). These shows that the modal order can be determined by $\Delta L_{i}$, further clarifying the critical modes.

$$
\begin{aligned}
& \Delta \mathbf{L}_{\mathbf{i}}= \frac{\int_{1} \varphi_{\text {POI }}(\mathbf{x}, \mathbf{y})_{\mathbf{i}}\left\{\mathbf{b}_{\mathbf{m i}}\right\}\{\mathbf{u}\}}{2 \zeta_{\mathbf{i}} \omega_{\mathbf{i}}{ }^{2}}, \\
&= \frac{\int_{1} \varphi_{\text {POI }}(\mathbf{x}, \mathbf{y})_{\mathbf{i}}\left[\Phi_{\mathbf{i}}\right]^{\mathbf{T}}[\mathbf{B}]\{\mathbf{u}\}}{2 \zeta_{\mathbf{i}} \omega_{\mathbf{i}}{ }^{2}} \\
& \Delta \mathbf{L}_{\mathbf{i}} \propto\left(\mathbf{p}_{\mathbf{f}}\right)_{\mathbf{i}} * \frac{\int_{1} \varphi_{\text {POI }}(\mathbf{x}, \mathbf{y})_{\mathbf{i}}}{2 \zeta_{\mathbf{i}} \omega_{\mathbf{i}}{ }^{2}},
\end{aligned}
$$

where $\left[\Phi_{i}\right]^{T}[B]\{u\}$ is the $i$ th modal force, $\left[\Phi_{i}\right]^{T}$ is the $i$ th transposition of the eigenvalue of the applied force point, $[B]$ is the force distribution matrix, $\{u\}$ is the input force amplitude; $\zeta_{i}$ and $\omega_{i}$ are the $i$ th damping ratio and eigenvalue, respectively; the sum of the $\phi \cdot P O I$ is determined by the position $(x, y)$ and the length $l$ of the optical fiber sensor.

(2) Determination of optimization goal

There are $n f$ alternative optimization locations; each has $m m$ nodes in the discrete structure. The square of the strain eigenvalue is taken as the optimization target from a strain energy perspective. And considering the effect of the force, the optimal position and length ordering of the critical modes are obtained: 


$$
\begin{aligned}
\mathbf{E}_{\mathbf{i}} & =\max \left(\left(\mathbf{p}_{\mathbf{f}}\right)_{\mathbf{i}} * \mathbf{E}_{\mathbf{i}}(\mathbf{x}, \mathbf{y}, \mathbf{l})\right) \\
& =\max \left[\left(\left(\mathbf{p}_{\mathbf{f}}\right)_{\mathbf{i}} * \sum_{\mathbf{j}_{\mathbf{j}=1}}^{\mathbf{m m}}(-1)_{\mathbf{i}} *\left(\phi_{\mathbf{s}}\left(\mathbf{x}_{\mathbf{j} \mathbf{j}}, \mathbf{y}_{\mathbf{j} \mathbf{j}}\right)\right)^{2}\right)\right],
\end{aligned}
$$

where $E_{i}$ is the $i$ th optimization target; $l$ is the length of the optical fiber sensor.

In the measurement, the measurement result of each sensor depends not only on the sensor layout but also on the measurement properties of the sensor itself. The measurement variance $\boldsymbol{\sigma}_{j}^{\mathbf{s}}$ of each sensor is taken as a correction factor to be added to the optimization target. If there are $n c$ sensors, the correction coefficient of the $j$ th sensor is as follows:

$$
\begin{gathered}
\boldsymbol{\alpha}_{\mathbf{j}}=\left(\frac{1}{\boldsymbol{\sigma}^{\mathbf{s}}}\right) / \sum_{\mathbf{j}=1}^{\mathbf{n c}}\left(\frac{1}{\boldsymbol{\sigma}^{\mathbf{s}}}\right), \\
\boldsymbol{\gamma}_{\mathbf{j}}=\frac{\boldsymbol{\alpha}_{\mathbf{j}}}{\max \left\{\boldsymbol{\alpha}_{1}, ., . ., \boldsymbol{\alpha}_{\mathbf{j}}, ., ., \boldsymbol{\alpha}_{\mathbf{n}}\right\}},
\end{gathered}
$$

where $\gamma_{j}$ is the correction coefficient of the $j$ th sensor.

Setting the proportion of the position and length of the sensor as $\mathrm{y}$ and the proportion of the property of the sensor itself as $(1-\mathfrak{y})$, the final correction coefficient of the sensor where the $i$ th critical mode is located is:

$$
\boldsymbol{\kappa}_{\mathbf{i}}=\mathfrak{\eta}+(1-\mathfrak{\eta}) \boldsymbol{\gamma}_{\mathbf{j}}
$$

The corrected optimization goal is:

$$
\widehat{\mathbf{E}}_{\mathbf{i}}(\mathbf{x}, \mathbf{y}, \mathbf{l})=\kappa_{\mathbf{i}} \mathbf{E}_{\mathbf{i}}(\mathbf{x}, \mathbf{y}, \mathbf{l}) .
$$

In order to reduce the redundancy of the output information at the location of each sensor, the correlation is used as a judgment index. The weak correlation value of less than 0.4 is chosen as the threshold value. Assuming that the eigenvalue corresponding to the first-ranked critical mode is $Q_{1}$, the eigenvalue $Q_{2}$ corresponding to the location and length of the second-ranked critical mode is limited by $Q_{1}$. Then the location of the second critical mode that has been optimized is screened for the second optimization, as following:

$$
\boldsymbol{\rho}_{12}=\frac{\operatorname{Cov}\left(\mathbf{Q}_{1}, \mathbf{Q}_{2}\right)}{\sqrt{\mathbf{Q}_{1}} \sqrt{\mathbf{Q}_{2}}}<0.4 .
$$

And so on, for the subsequent $i$ th order critical modes, the following conditions should be to be satisfied:

$$
\boldsymbol{\rho}_{1 \mathbf{i}} \& \boldsymbol{\rho}_{2 \mathbf{i}} \& \boldsymbol{\rho}_{3 \mathbf{i}} \& \ldots<0.4
$$

The optimal location and length of the optical fiber sensor corresponding to the critical modes can be obtained in turn through the above optimization.

The signal of the optimal sensor corresponding to each order of the flexible mode inevitably contains the information of the other orders, so the information transmitted by the sensors has a certain redundancy. On the other hand, the multiple sensors can reduce the uncertainty of measurement. Differential entropy can quantify the total uncertainty in the probability distribution of continuous random variables. The smaller the value is, the smaller the uncertainty is. Therefore, the following is proved from the perspective of differential entropy.

Assuming that $\Delta L$ conforms to a Gaussian distribution, the possibility that $n c(n c \geq 2)$ sensors will occur simultaneously is to multiply the probability of $n c$ events.

Let $N_{i}(\Delta L)$ be Gaussian PDFs with arbitrary mean $\mu_{i}$ and standard deviation $\sigma_{i}$,

$$
\left\{\begin{array}{l}
\mathbf{N}_{1}\left(\Delta \mathbf{L}, \boldsymbol{\mu}_{1}, \boldsymbol{\sigma}_{1}\right)=\frac{1}{\boldsymbol{\sigma}_{1} \sqrt{2 \pi}} \mathbf{e}^{-\frac{\left(\Delta \mathbf{L}-\boldsymbol{\mu}_{1}\right)^{2}}{2 \boldsymbol{\sigma}_{1}^{2}}} \\
\cdot \\
\mathbf{N}_{\mathbf{n c}}\left(\Delta \mathbf{L}, \boldsymbol{\mu}_{\mathbf{n}}, \boldsymbol{\sigma}_{\mathbf{n}}\right)=\frac{1}{\boldsymbol{\sigma}_{\mathbf{n}} \sqrt{2 \pi}} \mathbf{e}^{-\frac{\left(\Delta \mathbf{L}-\boldsymbol{\mu}_{\mathbf{n}}\right)^{2}}{2 \boldsymbol{\sigma}_{\mathbf{n}}{ }^{2}}}
\end{array}\right.
$$

Their product is:

$$
\begin{aligned}
& \mathbf{N}\left(\Delta \mathbf{L},\left(\mu_{1}, \sigma_{1}\right),\left(\mu_{2}, \sigma_{2}\right), ., ., .,\left(\mu_{\mathbf{n}}, \sigma_{\mathbf{n}}\right)\right)=\prod_{\mathbf{i}=1}^{\mathbf{n}} \frac{1}{\sigma_{\mathbf{i}} \sqrt{2 \pi \sigma_{\mathbf{i}}^{2}}} \mathbf{e}^{-\frac{\left(\Delta \mathbf{L}-\mu_{\mathbf{i}}\right)^{2}}{2 \sigma_{\mathbf{i}}{ }^{2}}} \\
& \Delta \mathbf{L}^{2}-2 \epsilon \frac{\sum_{\mathbf{j}=1}^{\mathbf{n c}}\left(\Delta \mathbf{L}_{\mathbf{j}} \prod_{\mathbf{i}=1}^{\mathbf{n c}(\mathbf{j} \not \subset \mathbf{n c})} \sigma_{\mathbf{i}}^{2}\right)}{\mathbf{n c}(\mathbf{n c}(\mathbf{j} \not \subset \mathbf{n c})}+\frac{\sum_{\mathbf{j}=1}^{\mathrm{nc}}\left(\Delta \mathbf{L}_{\mathbf{j}}^{2} \prod_{\mathbf{i}=1}^{\mathbf{n c}(\mathbf{j} \not \subset \mathbf{n c})} \sigma_{\mathbf{i}}^{2}\right)}{\mathbf{n c}(\mathbf{n c}(\mathbf{j} \not \subset \mathbf{n c})} \\
& \sum_{\mathbf{j}=1}^{\mathbf{n c}}\left(\prod_{\mathbf{i}=1}^{\mathbf{n c}(\mathbf{j} \not \mathbf{n c})} \sigma_{\mathbf{i}}^{2}\right) \quad \sum_{\mathbf{j}=1}^{\mathbf{n c}}\left(\prod_{\mathbf{i}=1}^{\mathbf{n c}(\mathbf{j} \not \subset \mathbf{n c})} \sigma_{\mathbf{i}}^{2}\right) \\
& 2 \prod^{\mathbf{n c}} \sigma_{\mathbf{i}}^{2} \\
& \frac{\mathbf{n} \mathbf{n} \mathbf{c}\left(\prod_{\mathbf{i}=1}^{\mathbf{n c}(\mathbf{j} \not \subset \mathbf{n c})} \sigma_{\mathbf{i}}^{2}\right)}{\sum^{\prime}} \\
& =\frac{1}{(2 \pi) \frac{\mathbf{n}}{2} \sqrt{\prod_{\mathbf{i}=1}^{\mathbf{n}} \sigma_{\mathbf{i}}^{2}}} \mathbf{e}
\end{aligned}
$$




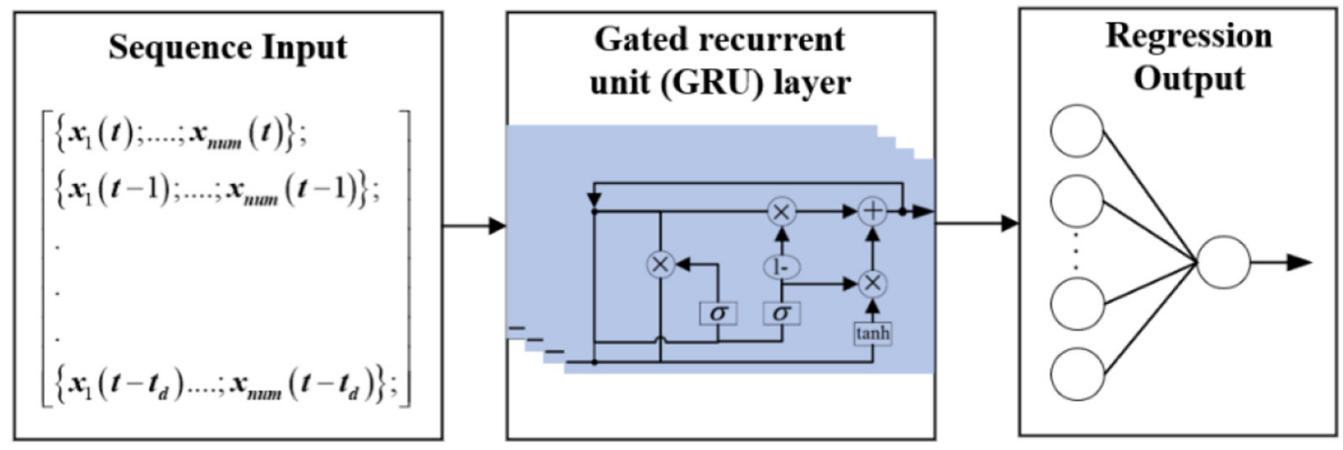

Fig. 3. Block diagram of the neural network structure.

Then the fusion standard deviation can be obtained as follows:

$$
\boldsymbol{\sigma}_{\text {fused }}^{2}=\frac{2 \prod_{\mathbf{i}=1}^{\mathbf{n c}} \boldsymbol{\sigma}_{\mathbf{i}}^{2}}{\sum_{\mathbf{j}=1}^{\mathbf{n c}}\left(\prod_{\mathbf{i}=1}^{\mathbf{n c}(\mathbf{j} \not \subset \mathbf{n})} \boldsymbol{\sigma}_{\mathbf{i}}^{2}\right)}=\boldsymbol{\sigma}_{\mathbf{j}}^{2}-\frac{\boldsymbol{\sigma}_{\mathbf{j}}^{4}\left(\sum_{\mathbf{i}=1}^{\mathbf{n c}(\mathbf{j} \not \subset \mathbf{n c})} \boldsymbol{\sigma}_{\mathbf{i}}^{2}\right)}{\sum_{\mathbf{j}=1}^{\mathbf{n c}}\left(\prod_{\mathbf{i}=1}^{\mathbf{n c}(\mathbf{j} \not \subset \mathbf{n c})} \boldsymbol{\sigma}_{\mathbf{i}}^{2}\right)}
$$

From the above derivation, it is clear that the variance of the fusion is smaller than that of any single sensor:

$$
\boldsymbol{\sigma}_{\text {fused }}^{2}<\boldsymbol{\sigma}_{\mathbf{j}}^{2}
$$

In terms of the differential entropy, it is derived as follows:

$$
\begin{aligned}
\mathbf{H}(\mathbf{x}) & =-\int \mathbf{N}(\Delta \mathbf{L}) \ln \mathbf{N}(\Delta \mathbf{L}) \mathbf{d} \Delta \mathbf{L} \\
& =\frac{\ln (\sqrt{2 \pi} \boldsymbol{\sigma})}{\boldsymbol{\sigma} \sqrt{2 \pi}} \sqrt{2} \boldsymbol{\sigma} \int \mathbf{e}^{-\frac{(\Delta \mathbf{L}-\boldsymbol{\mu})^{2}}{2 \boldsymbol{\sigma}^{2}}} \mathbf{d}\left(\frac{\Delta \mathbf{L}-\boldsymbol{\mu}}{\sqrt{2} \boldsymbol{\sigma}}\right) \\
+ & \frac{1}{\boldsymbol{\sigma} \sqrt{2 \pi}} \sqrt{2} \boldsymbol{\sigma} \int \mathbf{e}^{-\frac{(\Delta \mathbf{L}-\boldsymbol{\mu})^{2}}{2 \boldsymbol{\sigma}^{2}}} \frac{(\Delta \mathbf{L}-\boldsymbol{\mu})^{2}}{2 \boldsymbol{\sigma}^{2}} \mathbf{d}\left(\frac{\Delta \mathbf{L}-\boldsymbol{\mu}}{\sqrt{2} \boldsymbol{\sigma}}\right),
\end{aligned}
$$

Let $\mathbf{z}=\frac{\Delta \mathbf{L}-\boldsymbol{\mu}}{\sqrt{2} \mathbf{\sigma}}$ :

$$
\int_{-\infty}^{\infty} \mathbf{e}^{-\mathbf{z}^{2}} \mathbf{d z}=\sqrt{\boldsymbol{\pi}}
$$

Then the differential entropy becomes:

$$
\begin{aligned}
\mathbf{H}(\mathbf{x}) & =-\frac{\ln (\sqrt{2 \pi} \boldsymbol{\sigma})}{\boldsymbol{\sigma} \sqrt{2 \pi}} \sqrt{2} \boldsymbol{\sigma} \int_{-\infty}^{\infty} \mathbf{e}^{-\mathbf{z}^{2}} \mathbf{d} \mathbf{z} \\
& +\frac{1}{\boldsymbol{\sigma} \sqrt{2 \pi}} \sqrt{2} \boldsymbol{\sigma} \int_{-\infty}^{\infty} \mathbf{e}^{-\mathbf{z}^{2}} \mathbf{z}^{2} \mathbf{d} \mathbf{z} \\
& =\frac{1}{2}\left(\ln \left(2 \boldsymbol{\pi} \boldsymbol{\sigma}^{2}\right)+1\right) .
\end{aligned}
$$

Combining equations (21) and (24), it can be seen that multiple sensors can effectively reduce the uncertainty of measurement.

\subsection{POI displacement estimation model}

The displacement induced by flexibility is not instantaneous but a process of constant decay. Therefore, the current moment displacement is related to the displacement of the previous moments. Therefore, the constructed model needs to have dynamic properties. ANN is a nonlinear regression model and has been widely used for regression [30,31]. A neural network structure is constructed using the input layer with the input delays, a Gate Recurrent Unit (GRU) layer [32] and the output layer, as shown in Figure 3. The input of the input layer includes not only the current inputs but also the past inputs, while the GRU layer includes not only the output of the input layer but also the output of the hidden layer at the previous moments. These two layers involve the previous inputs, making the model dynamic. The POI model can be expressed:

$$
\widehat{\mathbf{y}}_{\mathbf{t}}=\mathbf{f}\left(\mathbf{x}_{\mathbf{t}}, \mathbf{x}_{\mathbf{t}-1}, \ldots, \mathbf{x}_{\mathbf{t}-\text { delay }}, \mathbf{w}_{1}, \mathbf{w}_{2}, \mathbf{w}_{3}, \mathbf{B}_{1}, \mathbf{B}_{2}, \mathbf{B}_{3}\right),
$$

where $w_{1}, w_{2}, w_{3}$ and $B_{1}, B_{2}, B_{3}$ represent the network weight vectors and the biases of the input-hidden layer, the hidden layer, and the hidden-output layer, respectively; $\widehat{\mathbf{y}}_{\mathbf{t}}$ stand for the estimated POI displacement.

The relative error $\left(\mathrm{E}_{\mathrm{H} 2}\right)$ is applied to estimate the accuracy of the estimated displacements intuitively.

$$
\mathbf{E}_{\mathbf{H}_{2}}=\frac{\left\|\mathbf{y}_{\text {ref }}-\widehat{\mathbf{y}}\right\|_{2}}{\left\|\mathbf{y}_{\text {ref }}\right\|_{2}} \times 100 \%,
$$

where $y_{r e f}$ is the reference POI displacement.

\section{Simulation analysis of functional optical fiber sensor optimization}

The simulation object, shown in Figure 4, is taken as the analysis object. In this figure, the central area is the interest area, and the flexible frequencies of interest within the $2500 \mathrm{~Hz}$ are the analysis focus. Through simulation analysis, the frequencies involved are $313 \mathrm{~Hz}, 851 \mathrm{~Hz}$, $1065 \mathrm{~Hz}, 1469 \mathrm{~Hz}, 1641 \mathrm{~Hz}$, and the corresponding order ranges from 7 th to 11 th. 


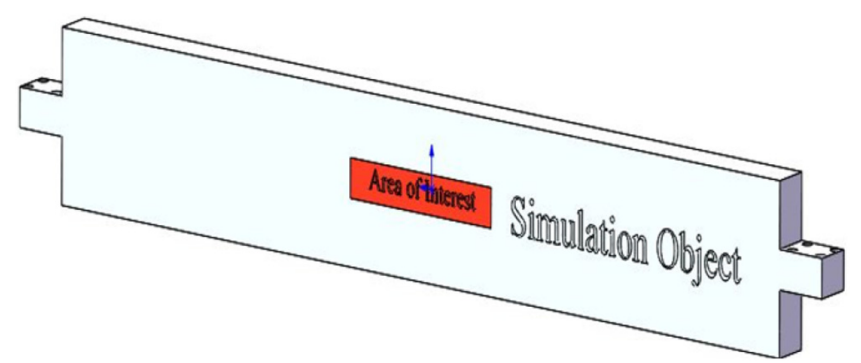

Fig. 4. Analyzed object with the area of interest.

Excluding the drive area of the analyzed object and other measurement areas, there are a total of three areas where the optical fiber sensors can be placed, each area being discretized into six locations, as shown in Figure 5. According to the constraints of the lengths and locations, there are 1275 choices to be selected for each location and a total of 19125 choices for the three regions.

According to the optimization method in Section 2, the critical modes of the desired region are first determined. Assuming a constant force input of $1 N$, the critical modes of the interest area are determined and ordered as $7 \mathrm{th}, 8 \mathrm{th}$, 11th, 9th, 10th. The critical modes become the 7 th mode and the 11th mode by adding the force constraint.

Since the measured sensor output information is not available, the correction coefficients of the sensors are set as:

$$
\left\{\begin{array}{l}
\boldsymbol{\kappa}_{1}=1 \\
\boldsymbol{\kappa}_{2}=1
\end{array} .\right.
$$

Then, the optimization target and correlation constraints are set based on the correlation. The optimization results and corresponding strain eigenvalue of the optical fiber sensors can be obtained for the 7th mode and the 11th mode, as shown in Table 1 and Figure 6, respectively.

\section{Experimental analysis}

To verify the role of the optical fiber sensor in estimating the POI displacement, an experimental device, as shown in Figure 7, was built according to the optical fiber measurement principle diagram in Section 2 and the sensor optimization results in Section 3. This experimental device mainly includes a set of capacitive sensors, optical fiber sensors and drive motors. The position and number of three sets of the capacitive sensors are assumed to be unchangeable. Sensors $1 \#$ and $3 \#$ are used for closed-loop control, and their solution results based on the rigid-body assumption are equivalent to the POC displacement; Sensor 2\# is a reference sensor, and its measurement result is equivalent to the POI displacement. The two sets of optical fiber sensors correspond to the 7th and 11th critical modes, respectively. Their measuring surface is on the same side as the measuring surface of the capacitive sensors. There are three sets of drive motors in total. Motors $1 \#$ and $3 \#$ are used for closed-loop control, and motor $2 \#$ is an excitation motor, which is used to excite the analysis object when the system is under closed-loop control. The data

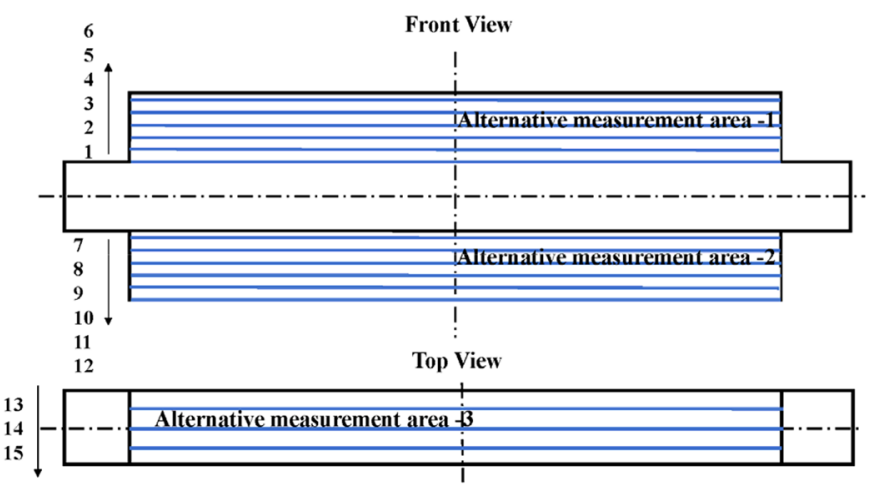

Fig. 5. Optical fiber sensor optimization - alternative areas.

acquisition for the capacitance sensors and the optical fiber sensors is performed using an AD board and a Zygo 4104c board with a sampling period of $5000 \mathrm{~Hz}$, respectively; the drive motor is driven by a linear driver VAREDAN-LA525.

\subsection{Correction of sensor optimization results}

According to the measurement principle diagram in Section 2, the two optical fiber sensor signals to be arranged were recorded without the active disturbance, as shown in Figure 8. The optical fiber sensors have high sensitivity and are susceptible to external disturbances, causing that the signal of the sensors have low-frequency drift. This low-frequency drift over time cannot characterize the measurement capability of the sensor. Therefore, the low-frequency drift needs to be eliminated. The signal was removed baseline drift based on the wavelet packet method $[33,34]$ with the wavelet basis function 'Db44' and the six-layer decomposition. After removing the lowfrequency drift, it can be observed in the histogram that the signal is normally distributed. So,

$$
\left\{\begin{array} { l } 
{ \boldsymbol { \sigma } _ { 1 } = 1 2 . 7 8 } \\
{ \boldsymbol { \sigma } _ { 2 } = 8 . 2 1 } \\
{ \boldsymbol { \sigma } _ { \text { fused } } = 7 . 1 9 }
\end{array} \Rightarrow \left\{\begin{array}{l}
\mathbf{H}\left(\boldsymbol{\sigma}_{1}\right)=3.97 \\
\mathbf{H}\left(\boldsymbol{\sigma}_{2}\right)=3.52 \\
\mathbf{H}\left(\boldsymbol{\sigma}_{\text {fused }}\right)=3.39
\end{array} .\right.\right.
$$

Based on the variances of the signals, it is known that

$$
\left\{\begin{array}{l}
\boldsymbol{\gamma}_{1}=0.6424 \\
\boldsymbol{\gamma}_{2}=1
\end{array}\right.
$$

The sensor properties are set to $20 \%$, then

$$
\left\{\begin{array}{l}
\boldsymbol{\kappa}_{1}=0.9285 \\
\boldsymbol{\kappa}_{2}=1
\end{array} .\right.
$$

Optical fiber sensor \#1 corresponds to the 7 th critical mode and Optical fiber sensor \#2 corresponds to the 11th critical mode. Then the corresponding eigenvalues after the correction are:

Comparing Figures 9 and 10, there is a certain effect on the magnitude of sensor $\# 1$ under the constraints of the sensor properties. However, it does not change the performance of the most critical modes on this sensor. If 
Table 1. Optical fiber sensor optimization result.

\begin{tabular}{llll}
\hline \multirow{2}{*}{ Modal order } & \multicolumn{2}{c}{ Optimal location } & Optimal length $/ \mathrm{mm}$ \\
\cline { 2 - 3 } & Location number & Position & 250 \\
7 & 11 & 925 & 390 \\
\hline
\end{tabular}

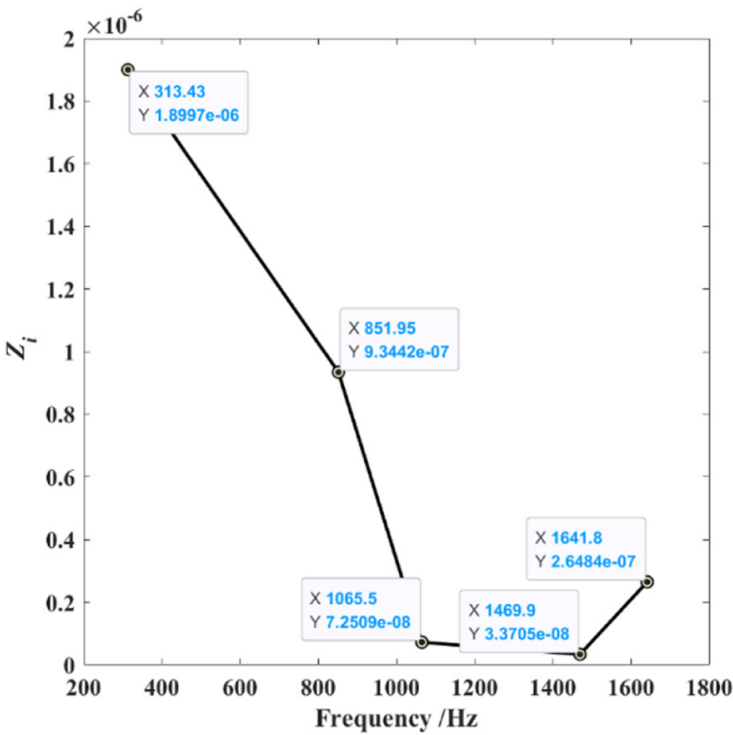

(a)

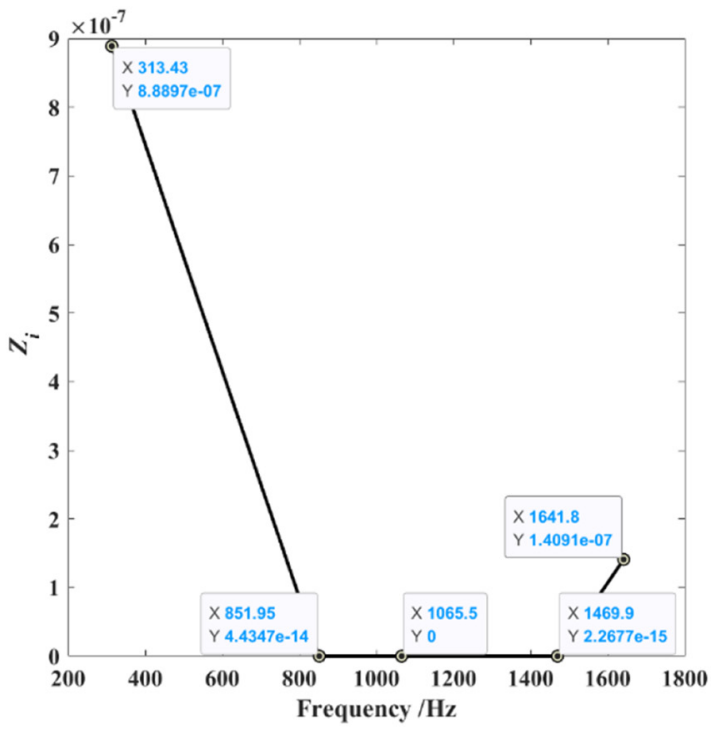

(b)

Fig. 6. Critical mode ordering: (a) under the force-free constraints; (b) under the force constraints.

the sensor properties affect the information acquisition of the mode, it is necessary to adjust the corresponding relationship with the sensors according to the order of the modes or to replace the sensor with the awful properties.

\subsection{Signal analysis}

The signals of the optical fiber sensors and the capacitive sensors are acquired under closed-loop control conditions from the host computer under a multi-sine sweep signal active disturbance outputting from motor $2 \#$. The spectrogram of the signals is illustrated in Figure 11.

Based on the extracted signals, the following conclusions are drawn.

- The actual analyzed object and the simulated object cannot be the same, so there is a certain gap between the obtained frequency. But this gap is not enough to affect the modal ordering for this structure in this paper.

- In the amplitude spectral density plot, the capacitive sensor output information contains flexible information; therefore, the POC displacement obtained by capacitive sensor \#1 and capacitive sensor \#2 based on the rigidbody assumption is not equal to the POI displacement of capacitive sensor $\# 2$.

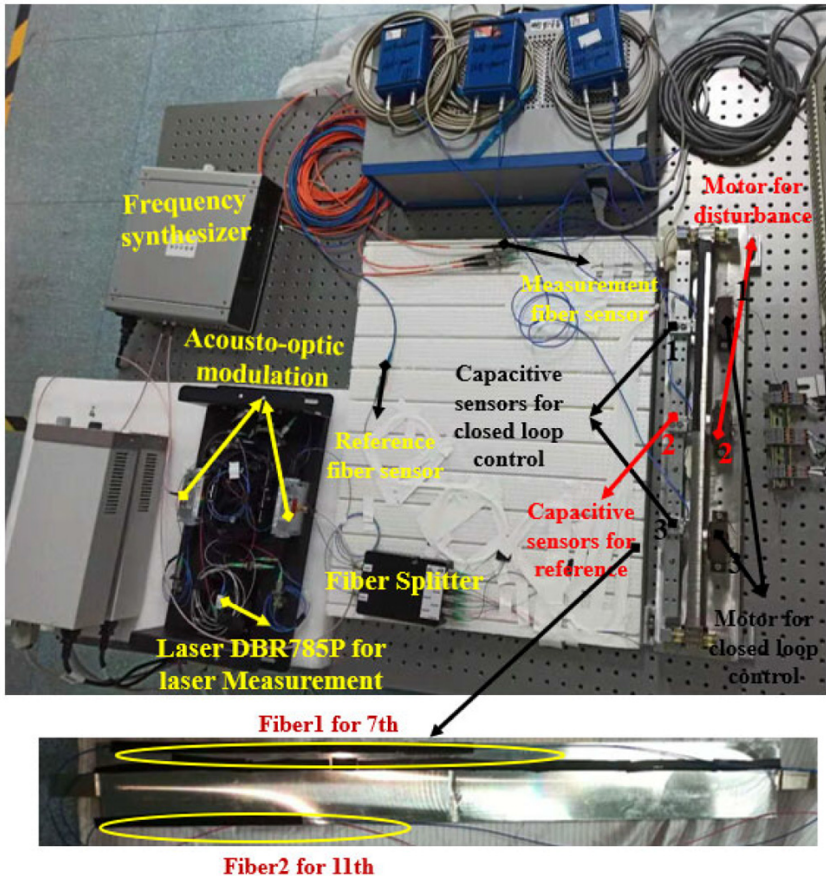

Fig. 7. Experimental setup. 


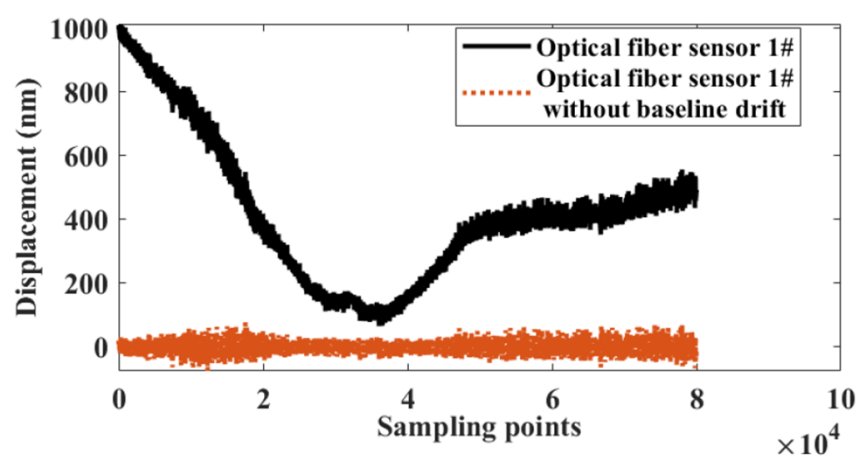

Histogram of the Optical Fiber Sensor 1\# without Baseline Drift

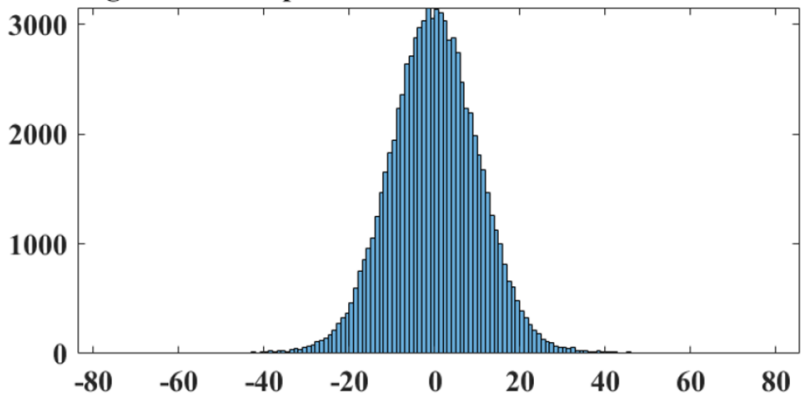

(a)
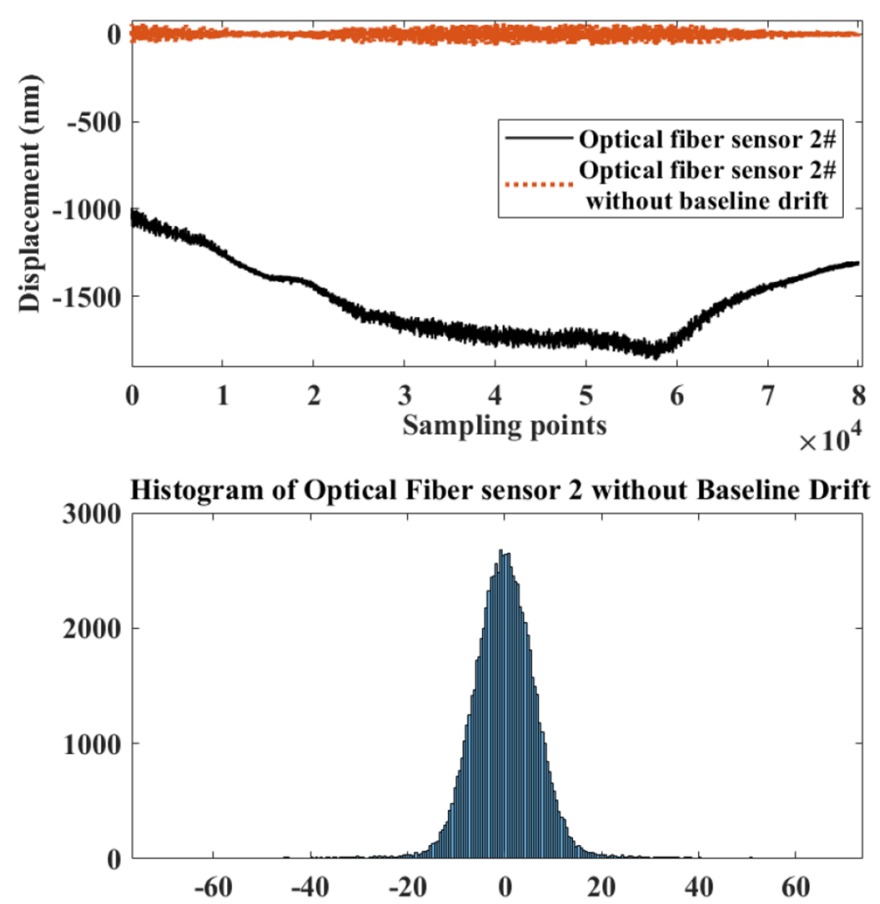

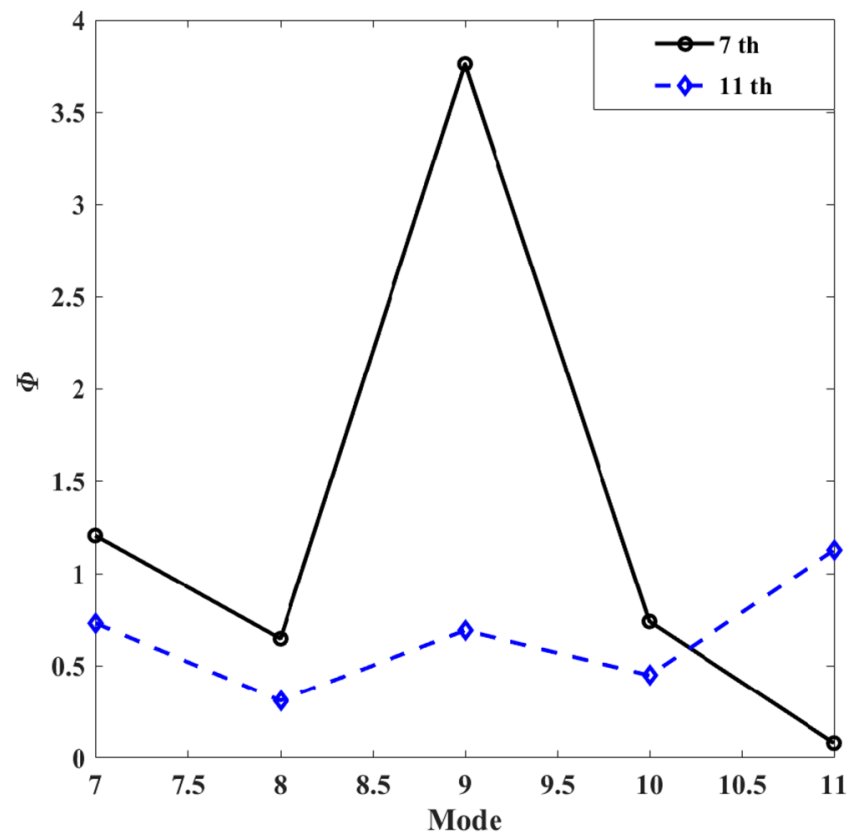

Fig. 9. Optimal result of optical fiber sensor: the optimal strain vector.

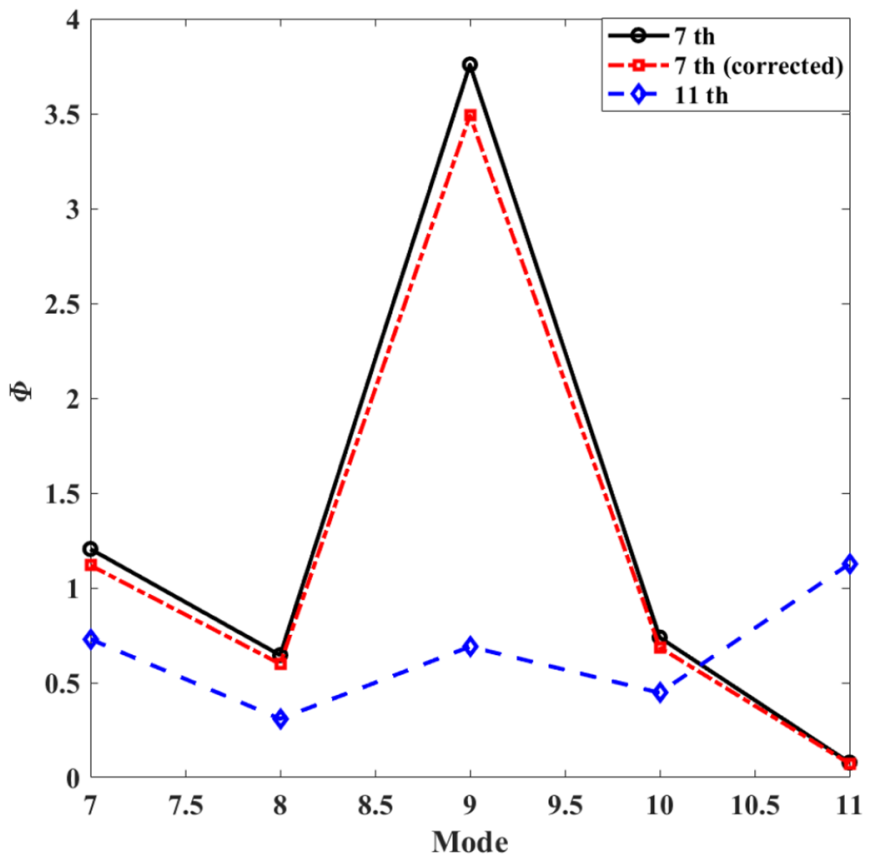

Fig. 10. Fiber optimization result: optimal strain vector. 

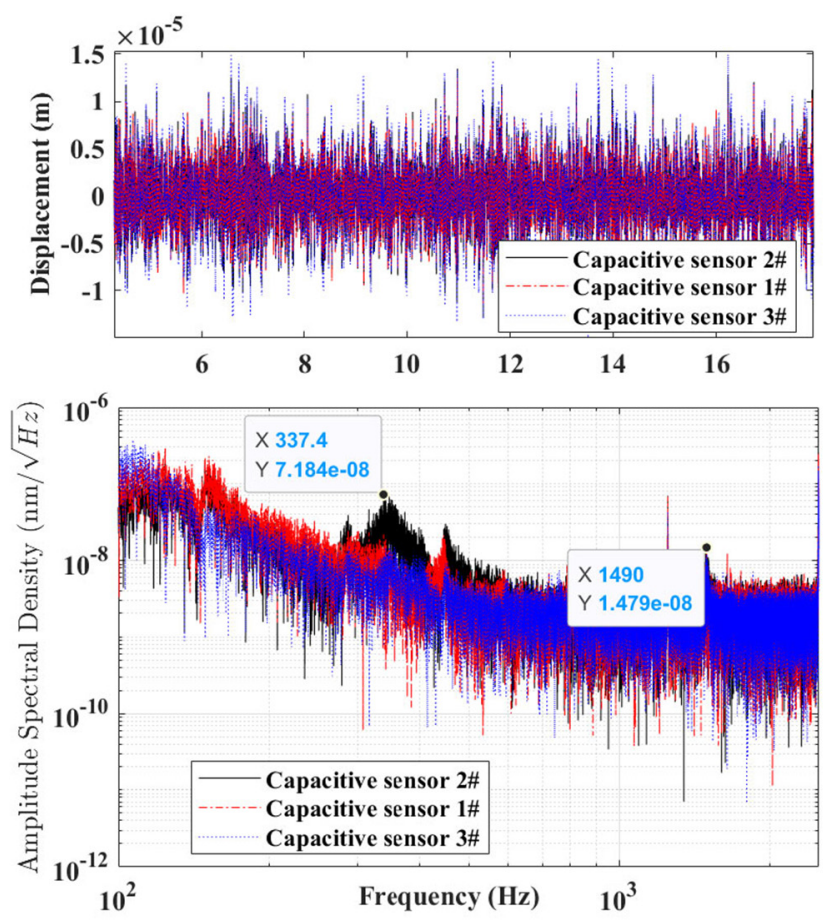

(a)
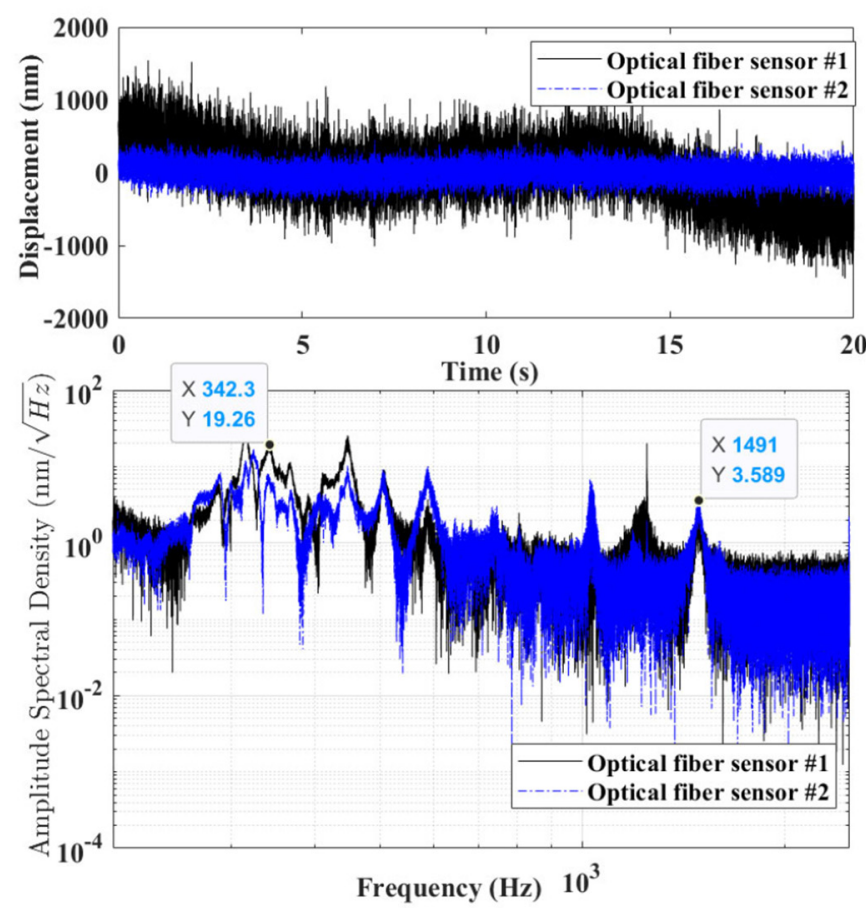

(b)

Fig. 11. Spectrogram of the signals: (a) capacitive sensors; (b) optical fiber sensors.

- Compared to optical fiber sensor \#2, optical fiber sensor \#1 has a higher amplitude in the low-frequency band and lower amplitude in the high-frequency band, being consistent with the optimized results of the optical fiber sensors in Section 3.

- The signals from the optical fiber sensors include all flexible information outputted by capacitive sensor \#2, while the flexible information output from capacitive sensor $\# 2$ is not reflected in the capacitive sensors \#1 and $\# 3$. In addition to the flexibility information of the measured object, the optical fiber sensors also contain additional flexible information belonging to the support base, being analyzed by the accelerometers, as shown in Figure 12. Compared to the more random signals in capacitive sensors $1 \#$ and $2 \#$, these deterministic signals can be easily separated from the original signals.

\subsection{POI displacement estimation model}

A POI displacement estimation model was constructed using the neural network structure mentioned in Section 2. This network structure includes five inputs with 60 delays and 512 GRU neurons. The five inputs are capacitive sensors $\# 1$ and $\# 3$, optical fiber sensors $\# 1$ and $\# 2$, and the exciting force. The excitation force is a complicated multi-sine excitation force with an excitation frequency of $10 \mathrm{~Hz} \sim 2000 \mathrm{~Hz}$. There are 10000 data sets collected in the experiment, being randomly separated into training and

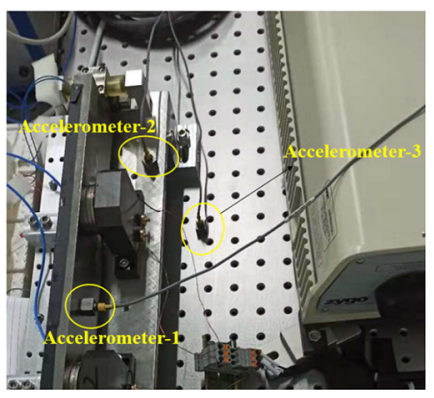

(a)

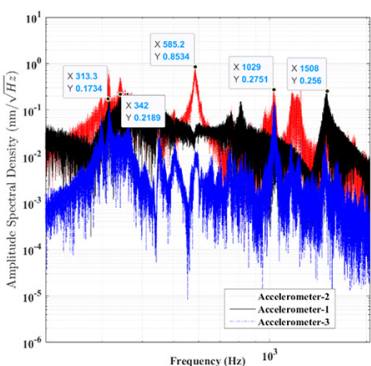

(b)
Fig. 12. Analysis of controlled objects by accelerometers: (a) experiment setup; (b) spectrogram of the signals.

validation sets as 70:30 ratios. Moreover, five other group data sets with 1000 data respectively are taken as the final test sample sets. The data are normalized into a range $[-1,1]$ to accelerate the convergence rate and improve the accuracy [35]. The training error is $0.9 \%$, while the five test errors are between $3.84 \%$ and $4.69 \%$; based on the rigid body assumption estimation method, the results of the five test groups are between $10.22 \%$ and $10.86 \%$. The average error of the five tests is shown in Figure 13. Therefore, the average estimation error of the proposed optical fiber sensor-based POI displacement estimation method is reduced by more than $61 \%$ compared with the rigid-body assumption-based estimation method. 


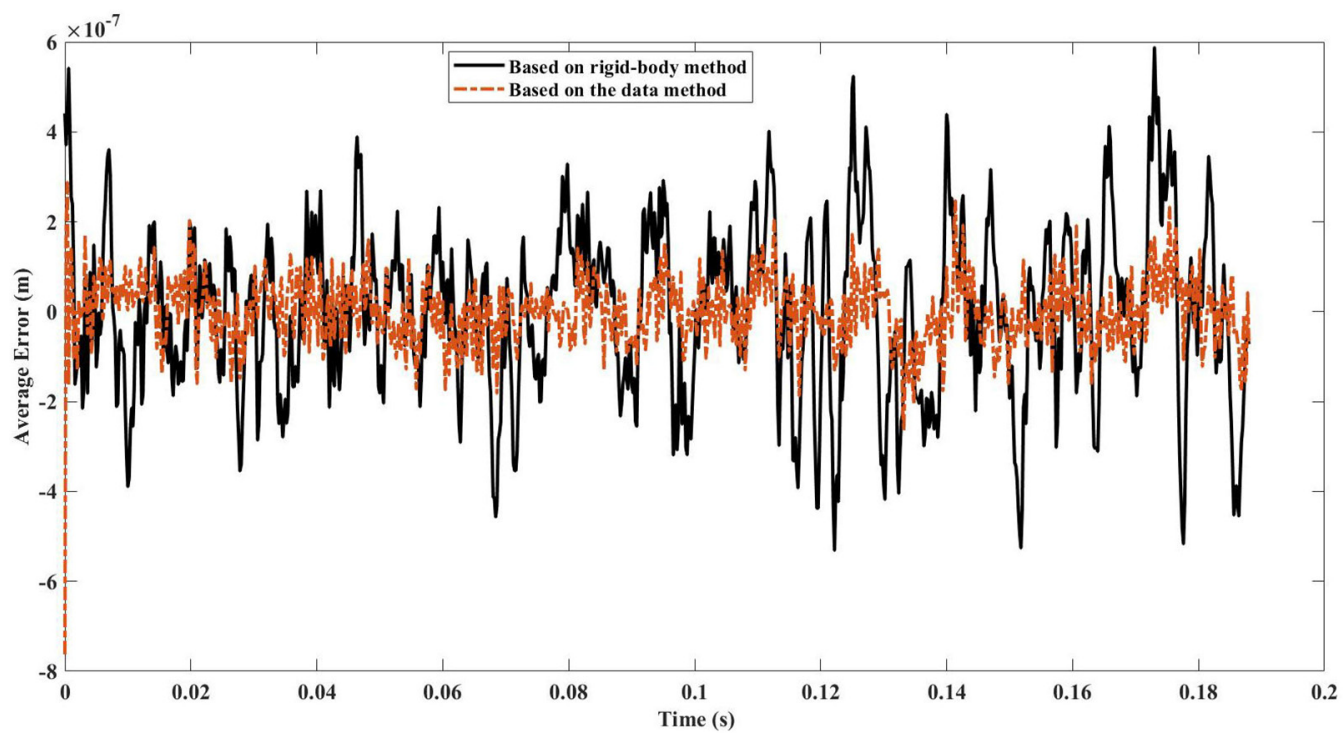

Fig. 13. Average error of the five group tests.

\section{Conclusion}

This paper proposes a POI displacement estimation method for ultra-precision motion systems based on the functional optical fiber sensor and the phase modulation principle. The core part of the paper is the measurement scheme and the layout optimization analysis based on the functional optical fiber sensor. To verify the effectiveness of the proposed estimation method, an experimental setup with two sets of measurement sensors, including capacitive sensors and optical fiber sensors, is designed. The following conclusions are drawn from the experimental analysis and the results of the POI displacement estimation based on the time-series dynamic model: (1) The analysis of the frequency domain plot of the test data shows that the output information of the optical fiber sensor contains more information about the flexible characteristics of the analyzed object than the output information of the capacitive sensors. (2) Through the estimation of the test data, the five groups of the test errors of the POI displacement estimation method using the optical fiber sensors proposed in this paper is between $3.84 \%$ and $4.69 \%$, while the errors are between $10.22 \%$ and $10.86 \%$ using the rigid-body assumption estimation method. Based on the latter error, the estimation error is reduced by about $61 \%$ compared with the rigid-body hypothesis-based estimation method.

This paper does not separate out the signals belonging to the controlled object in the optical fiber sensors when constructing the POI displacement estimation model. However, the valid and deterministic data can make the POI displacement estimation model built by the databased method have better generalization performance and estimation capability. In addition, there is not any detailed analysis of the model construction, such as the relationship between model complexity and model accuracy, and model generalization ability. Therefore, the specific model structure needs to be further optimized. In the next research work, we will focus on extracting the effective signal and constructing the best POI displacement estimation model to lay the foundation for subsequent applications.

\section{Disclosures}

The authors declare that there is no conflict of interest.

\section{References}

[1] T. Oomen et al., Next-generation wafer stage motion control: Connecting system identification and robust control, in: 2012 American Control Conference (ACC), Montreal, QC 2012, pp. 2455-2460

[2] M.J.C. Ronde, Feedforward control for lightweight motion systems, PhD thesis, Eindhoven University of Technology, 2014

[3] T. Oomen et al., Connecting system identification and robust control for next-generation motion control of a wafer stage, IEEE Trans. Control Syst. Technol. 22, 102-118 (2013)

[4] T. Oomen et al., Inferential motion control: Identification and robust control framework for positioning an unmeasurable point of interest, IEEE Trans. Control Syst. Technol. 23, 1602-1610 (2014)

[5] T. Oomen et al., Inferential motion control: Identification and robust control with unmeasured performance variables, in: 2011 50th IEEE Conference on Decision and Control and European Control Conference, Orlando, FL 2011, pp. 964-969

[6] M. Dorosti et al., Finite element model reduction and model updating of structures for control, IF AC Proc. 47, 4517-4522 (2014)

[7] G. Van Baars, Getting high tech systems in shape and fit for the future, Mikronie7k 52, 30-36 (2011) 
[8] Y. Jiang et al., Coupled dynamic modeling and analysis of ultra-precision 6-DOF fine stage, Proc. Chin. Soc. Electr. Eng. 30, 5451-5457 (2014)

[9] T. Oomen, E. Grassens, F. Hendriks, Inferential motion control: Identification and robust control framework for positioning an unmeasurable point of interest, IEEE Trans. Control Syst. Technol. 23, 1602-1610 (2014)

[10] J.G. Vogel et al., Estimation of the deformation of a plate using a limited number of sensors, in: ASPE Spring Topical Meeting MIT Laboratory for Manufacturing and Productivity Annual Summit, Cambridge, Massachusetts, 2013, pp. 21-23

[11] R.A.C.M. Beerens et al., Positioning system, lithographic apparatus and device manufacturing method, U.S. Patent, 9, $383,659[\mathrm{P}], 2016$

[12] T. Castenmiller et al., Towards ultimate optical lithography with NXT: 1950i dual stage immersion platform, Proc. SPIE 7640, Optical Microlithography XXIII, 76401N (2010)

[13] K.W. Verkerk, H. Butler, P.P.J. Van den Bosch, Improved disturbance rejection for high precision systems through estimation of the flexible modes[C], 2015 IEEE Conference on Control Applications (CCA), 1191-1196 (2015)

[14] R. Voorhoeve et al., Estimating structural deformations for inferential control: a disturbance observer approach, IFACPapersOnLine 49, 642-648 (2016)

[15] Mooren, et al., Compensating quasi-static disturbances for inferential control: an observer-based approach applied to a wafer stage, in: Proceedings of the 2018 IEEJ International Workshop on Sensing, Actuation, Motion Control, and Optimization Tokyo, Japan, 2018

[16] R. de Rozario et al., Identification of position-dependent mechanical systems, in: 35th Benelux Meeting on Systems and Control, Soesterberg, Netherlands, 2016 pp. 22-24

[17] M. Dorosti, Reduced-other model updating for prediction of performance variables in mechanical structures, $\mathrm{PhD}$ thesis, Eindhoven University of Technology, 2017

[18] R. Voorhoeve et al., Identifying position-dependent mechanical systems: A modal approach with applications to wafer stage control, arXiv preprint arXiv:1807.06942 (2018)

[19] S.H.J. Van der Kleij, Shape estimation of a compliant wafer chuck in lithography systems, Master thesis, Delft University of Technology, 2014

[20] A. Koevoets et al., Thermal-elastic compensation models for position control, in: Proceedings of the 24th ASPE Annual Meeting, United States, 2009
[21] T. Castenmiller, M.F.V.D., Towards ultimate optical lithography with NXT: 1950i dual stage immersion platform, Proc. SPIE 7640, 511-519 (2010)

[22] S. Cho, C. Yun, S. Sim, Displacement estimation of bridge structures using data fusion of acceleration and strain measurement incorporating finite element model, Smart Struct. Syst. 15, 645-663 (2015)

[23] S. Cho et al., Reference-free displacement estimation of bridges using kalman filter-based multimetric data fusion, J. Sens, 1-9 (2016)

[24] T. Örtel, J.F. Wagner, F. Saupe, Integrated motion measurement illustrated by a cantilever beam, Mech. Syst. Signal Process. 34, 131-145 (2013)

[25] D.A. Krohn, T. MacDougall, A. Mendez, Fiber Optic Sensors: Fundamentals and Applications, SPIE Press, Bellingham, WA, 2014

[26] R. Di Sante, Fiber optic sensors for structural health monitoring of aircraft composite structures: recent advances and applications, Sensors 15, 18666-18713 (2015)

[27] K.T.V. Grattan, B.T. Meggitt, L.S. Grattan, Optical Fiber Sensor Technology, Springer, 2000

[28] Y. Zhao, Principles and Application Technology of Optical Fiber Sensors, Tsinghua University Press, Beijing, 2007

[29] A. Dandridge, A.B. Tveten, T.G. Giallorenzi, Homodyne demodulation scheme for fiber optic sensors using phase generated carrier, IEEE J. Quant. Electr. 18, 1647-1653 (1982)

[30] Y. Yu, Research on Zygo Interferometer and Its Application, Master thesis, Tianjin University, 2005

[31] A. Prieto, B. Prieto, E.M. Ortigosa et al., Neural networks: An overview of early research, current frameworks and new challenges, Neurocomputing 214, 242-268 (2016)

[32] K. Cho et al., Learning phrase representations using RNN encoder-decoder for statistical machine translation, arXiv preprint arXiv:1406.1078 (2014)

[33] I. Daubechies, Ten Lectures on Wavelets, Society for Industrial and Applied Mat, 1992

[34] R. Yan, Base wavelet selection criteria for non-stationary vibration analysis in bearing health diagnosis, $\mathrm{PhD}$ thesis, University of Massachusetts Amherst, 2014

[35] J. Sola, J. Sevilla, Importance of input data normalization for the application of neural networks to complex industrial problems, IEEE Trans. Nucl. Sci. 44, 1464-1468 (1997)

Cite this article as: Y. Li, M. Zhang, Y. Zhu, Research on the estimation method of the point-of-interest (POI) displacement for ultra-precision flexible motion system based on functional optical fiber sensor, Mechanics \& Industry 22, 48 (2021) 